Proceedings of the

International Geometry Center

Vol. 10, no. 3-4 (2017) pp. 29-43

\title{
Інваріантні об'єкти конформно голоморфно-проективних перетворень ЛКК-многовидів
}

\author{
Є. В. Черевко, О. Є. Чепурна
}

\begin{abstract}
The article is devoted to the problem of holomorphically projective transformations of locally conformal Kähler manifolds. It's worth to be noted, that J. Mikes and Z. Radulovich have proved that a locally conformal Kähler manifold does not admit finite nontrivial holomorphically projective mappings for a Levi-Civita connection. Earlier we had also shown that a locally conformal Kähler manifold does not admit as well nontrivial infinitesimal holomorphically projective transformations for the Levi-Civita connection. But since the Weyl connection defined by Lee form on a locally conformal Kähler manifold is $F$-connection, hence for the connection nontrivial infinitesimal holomorphically projective transformations are possible.

In the present paper we rewrite the system of partial differential equations for the Levi-Civita connection and introduce so called infinitesimal conformal holomorphically projective transformations. This allows us to obtain necessary and sufficient conditions for a locally conformal Kähler manifold to have a group of infinitesimal conformal holomorphically projective transformations. We also calculate the number of parameters for such group, and describe tensor and non-tensor invariants preserved by group tansformations. Finally, we show that a vector field generating infinitesimal conformal holomorphically projective transformations of a compact locally conformal Kähler manifold is contravariant almost analytic.
\end{abstract}

Ключові слова: ермітові многовиди, конформно келерові мнговиди, форма Лі, конформно голоморфно-проективні перетворення. 


\begin{abstract}
Анотація. Статтю присвячено проблемі голоморфно-проективних перетворень. Й. Мікеш та Ж. Радулович показали, що локально конформнокелерові многовиди не дозволяють скінченних нетривіальних голоморфно проективних відображень для зв'язності Леві-Чівіта, а автори даної статті нещодавно довели, що ці многовиди не дозволяють також i нетривіальних інфінітезимальних голоморфно-проективних перетворень для зв'язності Леві-Чівіта. Але, оскільки зв'язність Вейля, що визначається на ЛКК-многовиді формою Лі, є $F$-зв'язністю, то нетривіальні інфінітезимальні голоморфно-проективні перетворення є можливими для неї. Якщо у такій системі диференціальних рівнянь у частинних похідних перейти до зв'зності Леві-Чівіта, то можна таким чином ввести конформно голоморфно-проективні перетворення.

В даній роботі отримано необхідні та достатні умови того, щоб локально конформно-келеровий многовид дозволяв нетривіальну групу конформно голоморфно проективних перетворень та обчислено максимальну кількість параметрів цієї групи. Також знайдено інваріантні об'єкти цих перетворень, один тензорного, другий нетензорного характеру, і доведено, що на компактному локально конформно-келеровому многовиді векторне поле, що генерує нетривіальні конформно голоморфно-проективні перетворення є контраваріантним майже аналітичним.
\end{abstract}

\title{
1. ВСтУП
}

Об'єктом дослідження в цій статті є локально конформно-келерові многовиди для яких $\operatorname{dim}\left(M^{n}\right)=n=2 m>2$. Конформно-келеровим многовидам присвячені роботи багатьох дослідників. Зокрема, локально конформно-келерові многовиди розглядались у роботах [4], [10], у монографії [1], голоморфно-проективні відображення многовидів досліджувалися у працях [12], [3]. Велику увагу інфінітезимальним голоморфно-проективним перетворенням майже комплексних многовидів приділено у монографії [6]. У роботі [13] доведено, що не існує інфінітезимальних голоморфно-проективних перетворень локально конформнокелерових многовидів у зв'язності Леві-Чівіта та введено конформно голоморфно-проективні перетворення. У цій роботі ми продовжуємо дослідження і отримуємо інваріантні об'єкти цих перетворень.

\section{2. ПОПЕРЕДНІ ВІДОМОСТІ}

2.1. ЛКК-многовиди. Спочатку дамо декілька необхідних означень.

Означення 2.2. Майже комплексною структурою $J$ називають такий aфiнор $J_{j}^{i}$, що:

$$
J_{\alpha}^{i} J_{j}^{\alpha}=-\delta_{j}^{i}
$$

Тут $\delta_{j}^{i}-$ символ Кронекера. 
Означення 2.3. Многовид, на якому задано майже комплексну структуру $J$, називають майже комплексним многовидом.

Означення 2.4. Майже комплексний многовид є майже ермітовим, якщо на ньому задано ермітову метрику:

$$
J_{i}^{\alpha} J_{j}^{\beta} g_{\alpha \beta}=g_{i j}
$$

Майже ермітовий многовид позначаємо $\left(M^{n}, J, g\right)$. Тут число $n \in$ дійсною розмірністю многовиду, $m$ - комплексною, тому $n=2 m$.

Означення 2.5. Майже ермітовий многовид $\left(M^{n}, J, g\right)$ є ермітовим, якщо майже комплексна структура є інтегровною [6].

Зауважимо, якщо майже комплексна структура $J$ та многовид $M^{n}$ будуть належати класу $C^{\omega}$, достатньою умовою інтегровності майже комплексної структури є тотожна рівність нулю тензора Нейєнхейса:

$$
N_{i j}^{k}=J_{i}^{\alpha}\left(\partial_{j} J_{\alpha}^{k}-\partial_{\alpha} J_{j}^{k}\right)-J_{j}^{\alpha}\left(\partial_{i} J_{\alpha}^{k}-\partial_{\alpha} J_{i}^{k}\right)=0
$$

або, що еквівалентно

$$
J_{i, j}^{k}=J_{i}^{\alpha} J_{j}^{\beta} J_{\alpha, \beta}^{k}
$$

Комою ми позначаємо коваріантну похідну у зв'язності, узгодженій з рімановою метрикою $g_{i j}$.

Якщо до того ж на ермітовому многовиді $\left(M^{n}, J, g\right)$ має місце

$$
J_{i, j}^{k}=0,
$$

то він є келеровим.

Означення 2.6. Ермітовий многовид $M^{n}$, має назву локалъно конформно-келерового (коротше, ЛКК-) многовидом, якщо існує відкрите покриття $\mathfrak{U}=\left\{U_{\alpha}\right\}$ многовиду $M$ та система $\Sigma=\left\{\sigma_{\alpha}: U_{\alpha} \rightarrow \mathbb{R}\right\}$ гладких функцій таких, що $\left\{\left.J\right|_{U_{\alpha}}, \hat{g}_{\alpha}=\left.e^{-2 \sigma_{\alpha}} g\right|_{U_{\alpha}}\right\}$ - келерова структура для будь якого $\alpha$. Перехід від метрики $\left.g\right|_{U_{\alpha}}$ до метрики $\left.e^{-2 \sigma_{\alpha}} g\right|_{U_{\alpha}}$ має назву локально конформного перетворення структури. Функція $\sigma$ має назву визначальної бункиї конформного перетворення [11].

Відомо, що на ЛКК-многовиді форма Лі (Lee form), компоненти якої визначаються формулою [9]

$$
\omega=\frac{1}{m-1} \delta \Omega \circ J \quad \text { або } \quad \omega_{i}=-\frac{2}{n-2} J_{\beta, \alpha}^{\alpha} J_{i}^{\beta},
$$

має бути замкненою:

$$
d \omega=0
$$


Коваріантну похідну майже комплексної структури ЛКК-многовиду можна знайти за формулою:

$$
J_{i, j}^{k}=\frac{1}{2}\left(\delta_{j}^{k} J_{i}^{\alpha} \omega_{\alpha}-\omega^{k} J_{i j}-J_{j}^{k} \omega_{i}+J_{\alpha}^{k} \omega^{\alpha} g_{i j}\right) .
$$

Iснує теорема [1, с. 2].

Теорема 2.7. Локальна зв'язність Леві-Чівіта $\hat{\nabla}^{\alpha}$ локальноӥ келерової метрики $\hat{g}_{\alpha}$ продовжуєтъся до глобально визначеної лінійної зв'язності без скруту

$$
\hat{\nabla}_{X} Y=\nabla_{X} Y-\frac{1}{2} \omega(X) Y-\frac{1}{2} \omega(Y) X+\frac{1}{2} g(X, Y) B
$$

для будв яких $X, Y \in \mathfrak{X}(M)$. Білъше того, є справедливим, що

$$
\hat{\nabla} g=\omega \otimes g \text {. }
$$

Зв'язність, що задано згідно (2.8) має назву зв'язності Bейля (Weyl connection) ЛKK-многовиду $\left(M^{n}, J, g\right)$. Зв'язність Вейля є майже комплексною або $F$-зв'язністю, тобто такою, що

$$
\hat{\nabla}_{X} J=0
$$

або у локальних координатах

$$
J_{i \mid j}^{k}=0
$$

\section{8. Інфінітезимальні дифеоморфізми многовидів.}

Означення 2.9. Перетворення многовиду $M^{n}$

$$
\bar{x}^{h}=x^{h}+\epsilon \xi^{h}\left(x^{1}, x^{2}, \ldots, x^{n}\right),
$$

де $\epsilon$ - довільний малий параметр незалежний від $x^{i}$ має назву $i н \oint i$ нітезимального перетворення многовиду $M^{n}$. Вектор $\xi\left(x^{1}, x^{2}, \ldots, x^{n}\right)$ має назву генератора перетворення.

Похідна Лi (Lie derivative) тензора $T_{j_{1} \ldots j_{q}}^{i_{1} \ldots i_{p}}$ типу $(p, q)$ уздовж векторного поля $\xi$ в координатах має вигляд [8, с. 196]:

$$
\begin{aligned}
\mathfrak{L}_{\xi} T_{j_{1} \ldots j_{q}}^{i_{1} \ldots i_{p}}= & T_{j_{1} \ldots j_{q}, s}^{i_{1} \ldots i_{p}} \xi^{s}+T_{k j_{2} \ldots j_{q}}^{i_{1} \ldots i_{p}} \xi_{, j_{1}}^{k}+\ldots+T_{j_{1} \ldots k}^{i_{1} \ldots i_{p}} \xi^{k}{ }_{, j_{q}}- \\
& -T_{j_{1} \ldots j_{q}}^{l i_{2} \ldots i_{p}} \xi^{i_{1}}{ }_{, l}-\ldots-T_{j_{1} \ldots j_{q}}^{i_{1} i_{2} \ldots l} \xi_{, l}^{i_{p}} .
\end{aligned}
$$

Зокрема, для похідної Лі метричного тензора $g$ отримуємо:

$$
\mathfrak{L}_{\xi} g_{i j}=\xi_{i, j}+\xi_{j, i}
$$


Якщо многовид $M^{n}$ зазнав перетворення, то метричний тензор $\bar{g}$ перетвореного $\bar{M}^{n}$ матиме вигляд [14, с. 275]

$$
\bar{g}_{i j}=g_{i j}+h_{i j} \epsilon \text {, }
$$

де $h_{i j}=\mathfrak{L}_{\xi} g_{i j}=\xi_{i, j}+\xi_{j, i}$. В той самий час, похідна Лі об'єкту зв'язності $\Gamma_{j k}^{h}$ може бути знайдена за формулою [5, с. 8]

$$
\mathfrak{L}_{\xi} \Gamma_{j k}^{h}=\xi_{, j k}^{h}+\xi^{m} R_{j m k}^{h} .
$$

Ми можемо у (2.14) опустити індекс $h$, згорнувши з $g_{h i}$ і таким чином отримати одне з центральних рівнянь теорії інфінітезимальних перетворень:

$$
\xi_{i, j k}=\xi_{\alpha} R_{k j i}^{\alpha}+g_{h i} \mathfrak{L}_{\xi} \Gamma_{j k}^{h} .
$$

Вигляд доданку $g_{h i} \mathfrak{L}_{\xi} \Gamma_{j k}^{h}$ залежатиме від конкретного типу інфінітезимальних перетворень. Нас цікавить випадок, коли векторне поле

$$
\xi\left(x^{1}, x^{2}, \ldots, x^{n}\right)
$$

породжує перетворення, що зберігає комплексну структуру [6]:

$$
\mathfrak{L}_{\xi} J_{j}^{i}=J_{j, k}^{i} \xi^{k}-J_{j}^{\alpha} \xi_{, \alpha}^{i}+J_{\alpha}^{i} \xi_{, j}^{\alpha}=0 .
$$

Таке поле має назву контраваріантного аналітичного векторного поля, а інфінітезимальне перетворення називають голоморфним. Варто зауважити, що оскільки дифференціювання Лі комутує з операцією зовнішнього дифференціювання

$$
d \mathfrak{L}_{\xi} \omega=\mathfrak{L}_{\xi} d \omega,
$$

то будь-які інфінітезимальні перетворення зберігають замкненість форми Лі.

\section{3. КОНФОРМНО ГОЛОМОРФНО-ПРОЕКТИВНІ ІНФІНІТЕЗИМАЛЬНІ ПЕРЕТВОРЕННЯ}

\section{1. Алгебра Лі векторних полів, що визначають конформно} голоморфно-проективні інфінітезимальні перетворення. Стосовно голоморфно-проективних інфінітезимальних перетворень, у роботі [13] було доведено теорему.

Теорема 3.2. ЛКК-многовид $M^{n}$, не дозволяе існування нетривіальних інфінітезимальних перетворень із збереженням комплексної структури та ї̈ коварінтної похідної у зв'язності Леві-Чівіта, зокрема maкux, що

$$
\mathfrak{L}_{\xi} \Gamma_{i j}^{h}=\rho_{j} \delta_{i}^{h}+\rho_{i} \delta_{j}^{h}-\rho_{t} J_{i}^{t} J_{j}^{h}-\rho_{t} J_{j}^{t} J_{i}^{h}
$$


У роботі [13] було запропоновано, користуючись ідеями, що викладено у [2], ввести інфінітезимальні конформно голоморфно-проективні перетворення ЛКК-многовидів. Система рівнянь, які на ЛКК-многовиді $\left(M^{n}, J, g\right)$ визначають конформно голоморфно-проективні інфінітезимальні перетворення із збереженням комплексної структури має вигляд:

$$
\begin{aligned}
\xi_{i, j}= & \xi_{i j}, \\
\rho_{, i}=\rho_{i}, & \\
\xi_{i, j k}= & \xi_{\alpha} R_{k j i}^{\alpha}+\frac{1}{2}\left(\left(\omega_{\alpha} \xi^{\alpha}\right)_{, k} g_{i j}+\left(\omega_{\alpha} \xi^{\alpha}\right)_{, j} g_{i k}-\left(\omega_{\alpha} \xi^{\alpha}\right)_{, i} g_{j k}-\right. \\
& \left.\quad-\omega_{i} \mathfrak{L}_{\xi} g_{j k}+\omega^{\alpha}\left(\mathfrak{L}_{\xi} g_{i \alpha}\right) g_{j k}\right)+ \\
& +\rho_{j} g_{i k}+\rho_{k} g_{i j}-\rho_{t} J_{j}^{t} J_{k i}-\rho_{t} J_{k}^{t} J_{j i}, \\
& \quad \frac{1}{2} \omega^{t} \rho_{t} g_{i j}-\frac{1}{2} \rho_{i} \omega_{j}-\frac{1}{2} \rho_{j} \omega_{i}+ \\
& +\frac{1}{n+2} \mathfrak{L}_{\xi}\left(R_{i j}-\frac{n-2}{2}\left(\omega_{i, j}+\frac{\omega_{i} \omega_{j}}{2}-\frac{\|\omega\|^{2} g_{i j}}{2}\right)-\frac{\Delta_{2} \omega g_{i j}}{2}\right), \\
\mathfrak{L}_{\xi} J_{j}^{i}= & \xi^{k} J_{j, k}^{i}-J_{j}^{\alpha} \xi_{, \alpha}^{i}+J_{\alpha}^{i} \xi_{, j}^{\alpha}=0,
\end{aligned}
$$

де $\|\omega\|^{2}=\omega_{i} \omega_{j} g^{i j}$ та $\Delta_{2} \omega=\omega_{i, j} g^{i j}$. Дійсно, оскільки на ЛКК-многовиді існує $F$-зв'язність Вейля, то у цій зв'язності можна визначити голоморфно-проективні перетворення. У відповідній системі рівняннь голоморфно-проективних перетворень у $F$-зв'язності Вейля ми перейшли до зв'язності Леві-Чівіта ЛКK-многовиду і таким чином отримали систему (3.1).

Нехай контраваріантний аналітичний вектор $\xi$ породжує інфінітезимальні конформно голоморфно-проективні перетворення на ЛККмноговиді $\left(M^{n}, g, J\right)$. Похідна Лі об'єкту зв'язності Леві-Чівіта матиме вигляд

$$
\begin{aligned}
& \mathfrak{L}_{\xi} \Gamma_{i j}^{h}=\frac{1}{2}\left(\delta_{j}^{h}\left(\mathfrak{L}_{\xi} \omega_{i}\right)+\delta_{i}^{h}\left(\mathfrak{L}_{\xi} \omega_{j}\right)\right.- \\
&\left.-g^{h r}\left(\mathfrak{L}_{\xi} \omega_{r}\right) g_{i j}-\omega^{h} \mathfrak{L}_{\xi} g_{i j}+g^{h \beta} \omega^{\alpha}\left(\mathfrak{L}_{\xi} g_{\beta \alpha}\right) g_{i j}\right)+ \\
& \quad+\rho_{j} \delta_{i}^{h}+\rho_{i} \delta_{j}^{h}-\rho_{t} J_{i}^{t} J_{j}^{h}-\rho_{t} J_{j}^{t} J_{i}^{h} .
\end{aligned}
$$

Ковектор $\rho$ з системи (3.1) має назву асоційованого ковектору до вектору $\xi$. Припустимо, що існують два контраваріантних аналітичних вектори $\xi$ та $\eta$, кожен з яких породжує інфінітезимальні конформно голоморфно-проективні перетворення. Їхніми асоційованими ковекторами нехай є відповідно $\rho$ та $\mu$. Причому, поле $\zeta$ є комутатором полів 
$\xi$ та $\eta$ :

$$
\zeta^{i}=[\xi, \eta]^{i}=\xi^{t} \partial_{t} \eta^{i}-\eta^{t} \partial_{t} \xi
$$

Відомо, що для будь-якого геометричного об'єкту $\Lambda$ є справедливою формула [6, p. 20]

$$
\mathfrak{L}_{[\xi, \eta]} \Lambda=\mathfrak{L}_{\xi} \mathfrak{L}_{\eta} \Lambda-\mathfrak{L}_{\eta} \mathfrak{L}_{\xi} \Lambda .
$$

У нашому випадку (3.3) дає

$$
\begin{aligned}
& \mathfrak{L}_{[\xi, \eta]} \Gamma_{i j}^{h}=\mathfrak{L}_{\xi} \mathfrak{L}_{\eta} \Gamma_{i j}^{h}-\mathfrak{L}_{\eta} \mathfrak{L}_{\xi} \Gamma_{i j}^{h}= \\
& =\frac{1}{2}\left(\delta_{j}^{h}\left(\mathfrak{L}_{\xi} \mathfrak{L}_{\eta} \omega_{i}\right)+\delta_{i}^{h}\left(\mathfrak{L}_{\xi} \mathfrak{L}_{\eta} \omega_{j}\right)-\right. \\
& \left.-g^{h r}\left(\mathfrak{L}_{\xi} \mathfrak{L}_{\eta} \omega_{r}\right) g_{i j}-\omega^{h} \mathfrak{L}_{\xi} \mathfrak{L}_{\eta} g_{i j}+g^{h \beta} \omega^{\alpha}\left(\mathfrak{L}_{\xi} \mathfrak{L}_{\eta} g_{\beta \alpha}\right) g_{i j}\right)+ \\
& +\mathfrak{L}_{\xi} \mu_{j} \delta_{i}^{h}+\mathfrak{L}_{\xi} \mu_{i} \delta_{j}^{h}-\mathfrak{L}_{\xi} \mu_{t} J_{i}^{t} J_{j}^{h}-\mathfrak{L}_{\xi} \mu_{t} J_{j}^{t} J_{i}^{h}- \\
& -\frac{1}{2}\left(\delta_{j}^{h}\left(\mathfrak{L}_{\eta} \mathfrak{L}_{\xi} \omega_{i}\right)+\delta_{i}^{h}\left(\mathfrak{L}_{\eta} \mathfrak{L}_{\xi} \omega_{j}\right)-\right. \\
& \left.-g^{h r}\left(\mathfrak{L}_{\eta} \mathfrak{L}_{\xi} \omega_{r}\right) g_{i j}-\omega^{h} \mathfrak{L}_{\eta} \mathfrak{L}_{\xi} g_{i j}+g^{h \beta} \omega^{\alpha}\left(\mathfrak{L}_{\eta} \mathfrak{L}_{\xi} g_{\beta \alpha}\right) g_{i j}\right)- \\
& -\mathfrak{L}_{\eta} \rho_{j} \delta_{i}^{h}-\mathfrak{L}_{\eta} \rho_{i} \delta_{j}^{h}+\mathfrak{L}_{\eta} \rho_{t} J_{i}^{t} J_{j}^{h}+\mathfrak{L}_{\eta} \rho_{t} J_{j}^{t} J_{i}^{h},
\end{aligned}
$$

тобто,

$$
\begin{aligned}
\mathfrak{L}_{[\xi, \eta]} \Gamma_{i j}^{h}= & \mathfrak{L}_{\zeta} \Gamma_{i j}^{h}=\frac{1}{2}\left(\delta_{j}^{h}\left(\mathfrak{L}_{\zeta} \omega_{i}\right)+\delta_{i}^{h}\left(\mathfrak{L}_{\zeta} \omega_{j}\right)-g^{h r}\left(\mathfrak{L}_{\zeta} \omega_{r}\right) g_{i j}-\right. \\
& \left.-\omega^{h} \mathfrak{L}_{\zeta} g_{i j}+g^{h \beta} \omega^{\alpha}\left(\mathfrak{L}_{\zeta} g_{\beta \alpha}\right) g_{i j}\right)+ \\
& +\theta_{j} \delta_{i}^{h}+\theta_{i} \delta_{j}^{h}-\theta_{t} J_{i}^{t} J_{j}^{h}-\theta_{t} J_{j}^{t} J_{i}^{h} .
\end{aligned}
$$

Тут $\theta_{i}=\mathfrak{L}_{\xi} \mu_{i}-\mathfrak{L}_{\eta} \rho_{i}-$ асоційований ковектор до поля $\zeta^{i}=[\xi, \eta]^{i} .3(3.4)$ ми бачимо, що $\zeta^{i}=[\xi, \eta]^{i}$ також породжує інфінітезимальні конформно голоморфно-проективні перетворення. Звідси випливає теорема.

Теорема 3.3. На ЛКК-многовидi $\left(M^{n}, g, J\right)$ множина контраваріантних аналітичних векторних полів, що породжують інфінітезимальні конформно голоморфно-проективні перетворення, утворюе алгебру תi.

Розглянемо випадок, коли конформно голоморфно-проективні перетворення є такими, що зберігають форму Лі, тобто

$$
\mathfrak{L}_{\xi} \omega_{i}=0 .
$$

У такому разі (3.2) матиме вигляд

$$
\begin{aligned}
\mathfrak{L}_{\xi} \Gamma_{i j}^{h}=\frac{1}{2} & \left(-\omega^{h} \mathfrak{L}_{\xi} g_{i j}+g^{h \beta} \omega^{\alpha}\left(\mathfrak{L}_{\xi} g_{\beta \alpha}\right) g_{i j}\right)+ \\
& +\rho_{j} \delta_{i}^{h}+\rho_{i} \delta_{j}^{h}-\rho_{t} J_{i}^{t} J_{j}^{h}-\rho_{t} J_{j}^{t} J_{i}^{h} .
\end{aligned}
$$


Якщо $\eta$ також є контраваріантним аналітичним векторним полем, що породжує конформно голоморфно-проективні перетворення, зберігаючи при цьому форму Лі

$$
\mathfrak{L}_{\eta} \omega_{i}=0
$$

то очевидно, що

$$
\mathfrak{L}_{[\xi, \eta]} \omega_{i}=0
$$

та

$$
\begin{gathered}
\mathfrak{L}_{[\xi, \eta]} \Gamma_{i j}^{h}=\frac{1}{2}\left(-\omega^{h} \mathfrak{L}_{[\xi, \eta]} g_{i j}+g^{h \beta} \omega^{\alpha}\left(\mathfrak{L}_{[\xi, \eta]} g_{\beta \alpha}\right) g_{i j}\right)+ \\
+\theta_{j} \delta_{i}^{h}+\theta_{i} \delta_{j}^{h}-\theta_{t} J_{i}^{t} J_{j}^{h}-\theta_{t} J_{j}^{t} J_{i}^{h},
\end{gathered}
$$

де коваріантні поля $\mu_{i}$ та $\theta_{i}=\mathfrak{L}_{\xi} \mu_{i}-\mathfrak{L}_{\eta} \rho_{i}$ є відповідно асоційованими до векторних полів $\eta_{i}$ та $\zeta^{i}=[\xi, \eta]^{i}$. Звідси випливає теорема.

Теорема 3.4. На ЛКK-многовидi $\left(M^{n}, g, J\right)$ множина контраваріантних аналітичних векторних полів, що породжують інфінітезимальні конформно голоморфно-проективні перетворення, зберігаючи форму $\pi i$

$$
\mathfrak{L}_{\xi} \omega_{i}=0
$$

утворюе алгебру Лi.

Зрозуміло, що ця алгебра є підалгеброю згаданої у попередній теоремі алгебри Лі контраваріантних аналітичних векторних полів, що породжують інфінітезимальні конформно голоморфно-проективні перетворення.

\section{5. Геометричні об'єкти, що є інваріантними при конформ-} но голоморфно-проективних інфінітезимальних перетвореннях. Згорнемо (3.2) за індексами $h$ та $j$.

$$
\mathfrak{L}_{\xi} \Gamma_{i s}^{s}=\frac{n}{2}\left(\mathfrak{L}_{\xi} \omega_{i}\right)+(n+2) \rho_{i} .
$$

Виразимо звідси асоційований ковектор $\rho_{i}$

$$
\rho_{i}=\frac{1}{n+2} \mathfrak{L}_{\xi} \Gamma_{i s}^{s}-\frac{n}{2(n+2)}\left(\mathfrak{L}_{\xi} \omega_{i}\right),
$$

та підставимо у (3.2)

$$
\begin{aligned}
\mathfrak{L}_{\xi} \Gamma_{i j}^{h}=\frac{1}{2}( & \delta_{j}^{h}\left(\mathfrak{L}_{\xi} \omega_{i}\right)+\delta_{i}^{h}\left(\mathfrak{L}_{\xi} \omega_{j}\right)- \\
& \left.\quad-g^{h r}\left(\mathfrak{L}_{\xi} \omega_{r}\right) g_{i j}-\omega^{h} \mathfrak{L}_{\xi} g_{i j}+g^{h \beta} \omega^{\alpha}\left(\mathfrak{L}_{\xi} g_{\beta \alpha}\right) g_{i j}\right)+ \\
& +\frac{1}{n+2}\left(\left(\mathfrak{L}_{\xi} \Gamma_{j s}^{s}-\frac{n}{2}\left(\mathfrak{L}_{\xi} \omega_{j}\right)\right) \delta_{i}^{h}+\left(\mathfrak{L}_{\xi} \Gamma_{i s}^{s}-\frac{n}{2}\left(\mathfrak{L}_{\xi} \omega_{i}\right)\right) \delta_{j}^{h}\right. \\
& \left.-\left(\mathfrak{L}_{\xi} \Gamma_{t s}^{s}-\frac{n}{2}\left(\mathfrak{L}_{\xi} \omega_{t}\right)\right) J_{i}^{t} J_{j}^{h}-\left(\mathfrak{L}_{\xi} \Gamma_{t s}^{s}-\frac{n}{2}\left(\mathfrak{L}_{\xi} \omega_{t}\right)\right) J_{j}^{t} J_{i}^{h}\right) .
\end{aligned}
$$


Розкриваючи дужки та зводячи подібні, отримуємо

$$
\begin{aligned}
\mathfrak{L}_{\xi} \Gamma_{i j}^{h} & +\frac{1}{2}\left(g^{h r}\left(\mathfrak{L}_{\xi} \omega_{r}\right) g_{i j}+\omega^{h} \mathfrak{L}_{\xi} g_{i j}-g^{h \beta} \omega^{\alpha}\left(\mathfrak{L}_{\xi} g_{\beta \alpha}\right) g_{i j}\right)- \\
& -\frac{1}{n+2}\left(\left(\mathfrak{L}_{\xi} \Gamma_{j s}^{s}+\left(\mathfrak{L}_{\xi} \omega_{j}\right)\right) \delta_{i}^{h}+\left(\mathfrak{L}_{\xi} \Gamma_{i s}^{s}+\left(\mathfrak{L}_{\xi} \omega_{i}\right)\right) \delta_{j}^{h}+\right. \\
& \left.+\left(\mathfrak{L}_{\xi} \Gamma_{t s}^{s}-\frac{n}{2}\left(\mathfrak{L}_{\xi} \omega_{t}\right)\right) J_{i}^{t} J_{j}^{h}+\left(\mathfrak{L}_{\xi} \Gamma_{t s}^{s}-\frac{n}{2}\left(\mathfrak{L}_{\xi} \omega_{t}\right)\right) J_{j}^{t} J_{i}^{h}\right)=0 .
\end{aligned}
$$

3 (3.5) ми бачимо, що об'єкт

$$
\begin{aligned}
\Pi_{i j}^{h}=\Gamma_{i j}^{h}+\frac{1}{2} \omega^{h} g_{i j}-\frac{1}{n+2} & \left(\left(\Gamma_{j s}^{s}+\omega_{j}\right) \delta_{i}^{h}+\left(\Gamma_{i s}^{s}+\omega_{i}\right) \delta_{j}^{h}+\right. \\
& \left.+\left(\Gamma_{t s}^{s}-\frac{n}{2} \omega_{t}\right) J_{i}^{t} J_{j}^{h}+\left(\Gamma_{t s}^{s}-\frac{n}{2} \omega_{t}\right) J_{j}^{t} J_{i}^{h}\right)
\end{aligned}
$$

є інваріантним під час інфінітезимальних конформно голоморфно-проективних перетворень.

Теорема 3.6. Якщо на ЛКК-многовидi $\left(M^{n}, g, J\right)$ контраварінтне аналітичне векторне поле $\xi$ породжуе інфінітезимальні конформно голоморфно-проективні перетворення, то об'єкт

$$
\begin{aligned}
\Pi_{i j}^{h}=\Gamma_{i j}^{h}+\frac{1}{2} \omega^{h} g_{i j}-\frac{1}{n+2} & \left(\left(\Gamma_{j s}^{s}+\omega_{j}\right) \delta_{i}^{h}+\left(\Gamma_{i s}^{s}+\omega_{i}\right) \delta_{j}^{h}+\right. \\
& \left.+\left(\Gamma_{t s}^{s}-\frac{n}{2} \omega_{t}\right) J_{i}^{t} J_{j}^{h}+\left(\Gamma_{t s}^{s}-\frac{n}{2} \omega_{t}\right) J_{j}^{t} J_{i}^{h}\right)
\end{aligned}
$$

є інваріантним відносно изих перетворень:

$$
\mathfrak{L}_{\xi} \Pi_{i j}^{h}=0 .
$$

Далі, знайдемо ще один інваріант. Існує тотожність [5, с. 16], яка стосовно метричного тензору $g$ набуває вигляду

$$
\left(\mathfrak{L}_{\xi} g_{i j}\right)_{, k}-\mathfrak{L}_{\xi} g_{i j, k}=g_{i s} \mathfrak{L}_{\xi} \Gamma_{k j}^{s}+g_{s j} \mathfrak{L}_{\xi} \Gamma_{k i}^{s} .
$$

Враховуючи рівняння (3.6) та те, що у випадку зв'язності Леві-Чівіта $g_{i j, k}=0$, матимемо:

$$
\begin{aligned}
\left(\mathfrak{L}_{\xi} g_{i j}\right)_{, k}= & \left(\omega_{\alpha} \xi^{\alpha}\right)_{, k} g_{i j}-\omega_{i} \mathfrak{L}_{\xi} g_{j k}+\omega^{\alpha}\left(\mathfrak{L}_{\xi} g_{i \alpha}\right) g_{j k}- \\
& -\omega_{j} \mathfrak{L}_{\xi} g_{i k}+\omega^{\alpha}\left(\mathfrak{L}_{\xi} g_{j \alpha}\right) g_{i k}+ \\
& +2 \rho_{k} g_{i j}+\rho_{j} g_{k i}+\rho_{i} g_{j k}-\rho_{t} J_{j}^{t} J_{k i}-\rho_{t} J_{i}^{t} J_{k j} .
\end{aligned}
$$

Далі, згідно [5, с. 17], для зв'язності Леві-Чівіта

$$
\mathfrak{L}_{\xi} R_{i j k}^{h}=\left(\mathfrak{L}_{\xi} \Gamma_{i k}^{h}\right)_{, j}-\left(\mathfrak{L}_{\xi} \Gamma_{i j}^{h}\right)_{, k} .
$$


Підставивши (3.2) в (3.8), отримуємо, що

$$
\begin{aligned}
& \mathfrak{L}_{\xi} R_{i j k}^{h}=\frac{1}{2}\left(\delta_{k}^{h}\left(\omega_{\alpha} \xi^{\alpha}\right)_{, i j}-g^{h r}\left(\omega_{\alpha} \xi^{\alpha}\right)_{, r j} g_{i k}-\right. \\
& \quad-\delta_{j}^{h}\left(\omega_{\alpha} \xi^{\alpha}\right)_{, i k}+g^{h r}\left(\omega_{\alpha} \xi^{\alpha}\right)_{, r k} g_{i j}- \\
&-\left(\omega^{h} \mathfrak{L}_{\xi} g_{i k}-g^{h \beta} \omega^{\alpha}\left(\mathfrak{L}_{\xi} g_{\beta \alpha}\right) g_{i k}\right)_{, j}+ \\
&+\left(\omega^{h} \mathfrak{L}_{\xi} g_{i j}-g^{h \beta} \omega^{\alpha}\left(\mathfrak{L}_{\xi} g_{\beta \alpha}\right) g_{i j}\right)_{, k}+ \\
&+ \rho_{i, j} \delta_{k}^{h}-\rho_{i, k} \delta_{j}^{h}- \\
&- \rho_{t, j} J_{i}^{t} J_{k}^{h}-\rho_{t} J_{i, j}^{t} J_{k}^{h}-\rho_{t} J_{i}^{t} J_{k, j}^{h}- \\
&-\rho_{t, j} J_{k}^{t} J_{i}^{h}-\rho_{t} J_{k, j}^{t} J_{i}^{h}-\rho_{t} J_{k}^{t} J_{i, j}^{h}+ \\
&+ \rho_{t, k} J_{i}^{t} J_{j}^{h}+\rho_{t} J_{i, k}^{t} J_{j}^{h}+\rho_{t} J_{i}^{t} J_{j, k}^{h}+ \\
&+ \rho_{t, k} J_{j}^{t} J_{i}^{h}+\rho_{t} J_{j, k}^{t} J_{i}^{h}+\rho_{t} J_{j}^{t} J_{i, k}^{h} .
\end{aligned}
$$

Тепер підставимо (3.1)4 в (3.9). Тоді використовуючи (2.7) та (3.7), матимемо

$$
\begin{aligned}
\mathfrak{L}_{\xi} R_{i j k}^{h}= & \frac{1}{2} \delta_{k}^{h} \mathfrak{L}_{\xi}\left(\omega_{i, j}+\frac{1}{2} \omega_{i} \omega_{j}-\frac{1}{2}\|\omega\|^{2} g_{i j}\right)- \\
- & \frac{1}{2} \delta_{j}^{h} \mathfrak{L}_{\xi}\left(\omega_{i, k}+\frac{1}{2} \omega_{i} \omega_{k}-\frac{1}{2}\|\omega\|^{2} g_{i k}\right)+ \\
+ & \frac{1}{2} \mathfrak{L}_{\xi}\left(\left(\omega^{h}{ }_{, k}+\frac{1}{2} \omega^{h} \omega_{k}\right) g_{i j}\right)-\frac{1}{2} \mathfrak{L}_{\xi}\left(\left(\omega^{h}{ }_{, j}+\frac{1}{2} \omega^{h} \omega_{j}\right) g_{i k}\right)+ \\
+\frac{1}{n+2}( & \delta_{k}^{h} \mathfrak{L}_{\xi}\left(R_{i j}-\frac{n-2}{2}\left(\omega_{i, j}+\frac{\omega_{i} \omega_{j}}{2}-\frac{\|\omega\|^{2} g_{i j}}{2}\right)-\frac{\Delta_{2} \omega g_{i j}}{2}\right) \\
& -\delta_{j}^{h} \mathfrak{L}_{\xi}\left(R_{i k}-\frac{n-2}{2}\left(\omega_{i, k}+\frac{\omega_{i} \omega_{k}}{2}-\frac{\|\omega\|^{2} g_{i k}}{2}\right)-\frac{\Delta_{2} \omega g_{i k}}{2}\right)+ \\
& +J_{k}^{h} J_{i}^{t} \mathfrak{L}_{\xi}\left(R_{t j}-\frac{n-2}{2}\left(\omega_{t, j}+\frac{\omega_{t} \omega_{j}}{2}-\frac{\|\omega\|^{2} g_{t j}}{2}\right)-\frac{\Delta_{2} \omega g_{t j}}{2}\right)- \\
& -J_{j}^{h} J_{i}^{t} \mathfrak{L}_{\xi}\left(R_{t k}-\frac{n-2}{2}\left(\omega_{t, k}+\frac{\omega_{t} \omega_{k}}{2}-\frac{\|\omega\|^{2} g_{t k}}{2}\right)-\frac{\Delta_{2} \omega g_{t k}}{2}\right)+ \\
& +J_{i}^{h} J_{j}^{t} \mathfrak{L}_{\xi}\left(R_{t k}-\frac{n-2}{2}\left(\omega_{t, k}+\frac{\omega_{t} \omega_{k}}{2}-\frac{\|\omega\|^{2} g_{t k}}{2}\right)-\frac{\Delta_{2} \omega g_{t k}}{2}\right)- \\
& \left.\quad-J_{i}^{h} J_{k}^{t} \mathfrak{L}_{\xi}\left(R_{t j}-\frac{n-2}{2}\left(\omega_{t, j}+\frac{\omega_{t} \omega_{j}}{2}-\frac{\|\omega\|^{2} g_{t j}}{2}\right)-\frac{\Delta_{2} \omega g_{t j}}{2}\right)\right) .
\end{aligned}
$$

Перегрупуємо та зведемо подібні доданки. В результаті матимемо, що 


$$
\begin{aligned}
& \mathfrak{L}_{\xi} R_{i j k}^{h}-\delta_{k}^{h} \mathfrak{L}_{\xi}\left(2 \omega_{i, j}+\omega_{i} \omega_{j}-\|\omega\|^{2} g_{i j}\right)+ \\
& +\delta_{j}^{h} \mathfrak{L}_{\xi}\left(2 \omega_{i, k}+\omega_{i} \omega_{k}-\|\omega\|^{2} g_{i k}\right)- \\
& -\frac{1}{2} \mathfrak{L}_{\xi}\left(\left(\omega^{h}{ }_{, k}+\frac{1}{2} \omega^{h} \omega_{k}\right) g_{i j}\right)+\frac{1}{2} \mathfrak{L}_{\xi}\left(\left(\omega^{h}{ }_{, j}+\frac{1}{2} \omega^{h} \omega_{j}\right) g_{i k}\right)- \\
& -\frac{1}{n+2}\left(\delta_{k}^{h} \mathfrak{L}_{\xi}\left(R_{i j}-\frac{\Delta_{2} \omega g_{i j}}{2}\right)-\delta_{j}^{h} \mathfrak{L}_{\xi}\left(R_{i k}-\frac{\Delta_{2} \omega g_{i k}}{2}\right)+\right. \\
& \quad+\left(J_{k}^{h} J_{i}^{t}-J_{i}^{h} J_{k}^{t}\right) \mathfrak{L}_{\xi}\left(R_{t j}-\frac{\Delta_{2} \omega g_{t j}}{2}-\right. \\
& \left.\quad-\frac{n-2}{2}\left(\omega_{t, j}+\frac{\omega_{t} \omega_{j}}{2}-\frac{\|\omega\|^{2} g_{t j}}{2}\right)\right)- \\
& \quad-\left(J_{j}^{h} J_{i}^{t}-J_{i}^{h} J_{j}^{t}\right) \mathfrak{L}_{\xi}\left(R_{t k}-\frac{\Delta_{2} \omega g_{t k}}{2}-\right. \\
& \left.\left.-\frac{n-2}{2}\left(\omega_{t, k}+\frac{\omega_{t} \omega_{k}}{2}-\frac{\|\omega\|^{2} g_{t k}}{2}\right)\right)\right)=0 .
\end{aligned}
$$

3 (3.10) ми бачимо, що тензор

$$
\begin{aligned}
P_{i j k}^{h} & =R_{i j k}^{h}-\delta_{k}^{h}\left(2 \omega_{i, j}+\omega_{i} \omega_{j}-\|\omega\|^{2} g_{i j}\right)+ \\
& +\delta_{j}^{h}\left(2 \omega_{i, k}+\omega_{i} \omega_{k}-\|\omega\|^{2} g_{i k}\right)- \\
& -\frac{1}{2}\left(\omega_{, k}^{h}+\frac{1}{2} \omega^{h} \omega_{k}\right) g_{i j}+\frac{1}{2}\left(\omega^{h}{ }_{, j}+\frac{1}{2} \omega^{h} \omega_{j}\right) g_{i k}- \\
& -\frac{1}{n+2}\left(\delta_{k}^{h}\left(R_{i j}-\frac{\Delta_{2} \omega g_{i j}}{2}\right)-\delta_{j}^{h}\left(R_{i k}-\frac{\Delta_{2} \omega g_{i k}}{2}\right)+\right. \\
& +\left(J_{k}^{h} J_{i}^{t}-J_{i}^{h} J_{k}^{t}\right)\left(R_{t j}-\frac{n-2}{2}\left(\omega_{t, j}+\frac{\omega_{t} \omega_{j}}{2}-\frac{\|\omega\|^{2} g_{t j}}{2}\right)-\frac{\Delta_{2} \omega g_{t j}}{2}\right)- \\
& \left.-\left(J_{j}^{h} J_{i}^{t}-J_{i}^{h} J_{j}^{t}\right)\left(R_{t k}-\frac{n-2}{2}\left(\omega_{t, k}+\frac{\omega_{t} \omega_{k}}{2}-\frac{\|\omega\|^{2} g_{t k}}{2}\right)-\frac{\Delta_{2} \omega g_{t k}}{2}\right)\right)
\end{aligned}
$$

зберігається при інфінітезимальних конформно голоморфно-проективних перетвореннях. Таким чином, ми отримали теорему.

Теорема 3.7. Якщо на ЛКК-многовидi $\left(M^{n}, g, J\right)$ контраварінтне аналітичне векторне поле $\xi$ породжуе інфінітезимальні конформно голоморбно-проективні перетворення, то тензор (3.11) е інваріантним відносно перетворенъ

$$
\mathfrak{L}_{\xi} P_{i j k}^{h}=0 .
$$

Зауважимо, що (3.12) - це скорочена форма (3.10), яка є умовою інтегровності рівняння $(3.1)_{3}$. Знайдемо умови інтегровності рівняння $(3.1)_{4}$. Диференціюючи коваріантно $(3.1)_{4}$ по $x^{k}$ та альтернуючи, маємо: 


$$
\begin{aligned}
\rho_{i, j k}-\rho_{i, j k} & = \\
=\frac{1}{n+2} & \left(\left(\mathfrak{L}_{\xi}\left(R_{i j}-\frac{n-2}{2}\left(\omega_{i, j}+\frac{\omega_{i} \omega_{j}}{2}-\frac{\|\omega\|^{2} g_{i j}}{2}\right)-\frac{\Delta_{2} \omega g_{i j}}{2}\right)\right)_{, j}-\right. \\
- & \left.\left(\mathfrak{L}_{\xi}\left(R_{i k}-\frac{n-2}{2}\left(\omega_{i, k}+\frac{\omega_{i} \omega_{k}}{2}-\frac{\|\omega\|^{2} g_{i k}}{2}\right)-\frac{\Delta_{2} \omega g_{i k}}{2}\right)\right)_{, k}\right)+ \\
& +\frac{1}{2} \omega_{t, k} \rho^{t} g_{i j}+\frac{1}{2} \omega^{t} \rho_{t, k} g_{i j}-\frac{1}{2} \omega_{j} \rho_{i, k}-\frac{1}{2} \omega_{i, k} \rho_{j}- \\
& \quad-\frac{1}{2} \omega_{t, j} \rho^{t} g_{i k}-\frac{1}{2} \omega^{t} \rho_{t, j} g_{i k}+\frac{1}{2} \omega_{k} \rho_{i, j}+\frac{1}{2} \omega_{i, j} \rho_{k}=\rho_{t} R_{i j k}^{t} .
\end{aligned}
$$

Враховуючи знову $(3.1)_{4}$, отримуємо

$$
\begin{aligned}
\rho_{t} P_{i j k}^{t}=\frac{1}{n+2} \mathfrak{L}_{\xi} & \left(\left(R_{i j}-\frac{n-2}{2}\left(\omega_{i, j}+\frac{\omega_{i} \omega_{j}}{2}-\frac{\|\omega\|^{2} g_{i j}}{2}\right)-\frac{\Delta_{2} \omega g_{i j}}{2}\right)_{, k}-\right. \\
- & \left(R_{i k}-\frac{n-2}{2}\left(\omega_{i, k}+\frac{\omega_{i} \omega_{k}}{2}-\frac{\|\omega\|^{2} g_{i k}}{2}\right)-\frac{\Delta_{2} \omega g_{i k}}{2}\right)_{, j}+ \\
+ & \frac{1}{2}\left(\delta_{i}^{t} \omega_{k}+\delta_{i}^{t} \omega_{k}-\omega^{t} g_{i k}\right) \times \\
& \times\left(R_{t j}-\frac{n-2}{2}\left(\omega_{t, j}+\frac{\omega_{t} \omega_{j}}{2}-\frac{\|\omega\|^{2} g_{t j}}{2}\right)-\frac{\Delta_{2} \omega g_{t j}}{2}\right)- \\
- & \frac{1}{2}\left(\delta_{i}^{t} \omega_{j}+\delta_{i}^{t} \omega_{j}-\omega^{t} g_{i j}\right) \times \\
& \left.\times\left(R_{t k}-\frac{n-2}{2}\left(\omega_{t, k}+\frac{\omega_{t} \omega_{k}}{2}-\frac{\|\omega\|^{2} g_{t k}}{2}\right)-\frac{\Delta_{2} \omega g_{t k}}{2}\right)\right),
\end{aligned}
$$

де $P_{i j k}^{t}$ є інваріантним тензором (3.11), який можна вважати аналогом тензору голоморфно-проективної кривини келерових многовидів. Диференціюючи декілька разів (3.10) та (3.13) ми можемо отримати диференціальні продовження умов інтегровності. Для зручності користуючись тотожністю для похідної Лі коваріантної похідної тензору [5, с.16], ми отримуємо перше продовження для (3.10):

$$
\mathfrak{L}_{\xi}\left(P_{i j k}^{h}\right)_{, l}=\mathfrak{L}_{\xi} \Gamma_{t l}^{h} P_{i j k}^{t}-\mathfrak{L}_{\xi} \Gamma_{i l}^{t} P_{t j k}^{h}-\mathfrak{L}_{\xi} \Gamma_{j l}^{t} P_{i t k}^{h}-\mathfrak{L}_{\xi} \Gamma_{k l}^{t} P_{i j t}^{h},
$$

де $\mathfrak{L}_{\xi} \Gamma_{j k}^{h}$ та $P_{i j k}^{h}$ визначені у рівняннях (3.2) та (3.11) відповідно. Аналогічно, можна знайти продовження для (3.13)

$$
\mathfrak{L}_{\xi}\left(P_{i j k}\right)_{, l}-\left(\mathfrak{L}_{\xi} P_{i j k}\right)_{, l}=-\mathfrak{L}_{\xi} \Gamma_{i l}^{t} P_{t j k}-\mathfrak{L}_{\xi} \Gamma_{j l}^{t} P_{i t k}-\mathfrak{L}_{\xi} \Gamma_{k l}^{t} P_{i j t}
$$

У (3.15) позначено

$$
\begin{aligned}
P_{i j k}= & \left(R_{i j}-\frac{n-2}{2}\left(\omega_{i, j}+\frac{\omega_{i} \omega_{j}}{2}-\frac{\|\omega\|^{2} g_{i j}}{2}\right)-\frac{\Delta_{2} \omega g_{i j}}{2}\right)_{, k}- \\
- & \left(R_{i k}-\frac{n-2}{2}\left(\omega_{i, k}+\frac{\omega_{i} \omega_{k}}{2}-\frac{\|\omega\|^{2} g_{i k}}{2}\right)-\frac{\Delta_{2} \omega g_{i k}}{2}\right)_{, j}+ \\
+ & \frac{1}{2}\left(\delta_{i}^{t} \omega_{k}+\delta_{i}^{t} \omega_{k}-\omega^{t} g_{i k}\right) \times \\
& \times\left(R_{t j}-\frac{n-2}{2}\left(\omega_{t, j}+\frac{\omega_{t} \omega_{j}}{2}-\frac{\|\omega\|^{2} g_{t j}}{2}\right)-\frac{\Delta_{2} \omega g_{t j}}{2}\right)- \\
- & \frac{1}{2}\left(\delta_{i}^{t} \omega_{j}+\delta_{i}^{t} \omega_{j}-\omega^{t} g_{i j}\right) \times
\end{aligned}
$$




$$
\times\left(R_{t k}-\frac{n-2}{2}\left(\omega_{t, k}+\frac{\omega_{t} \omega_{k}}{2}-\frac{\|\omega\|^{2} g_{t k}}{2}\right)-\frac{\Delta_{2} \omega g_{t k}}{2}\right) .
$$

Можна продовжувати диференціювання доки не виявиться, що на деякому етапі отримані рівняння задовольняються тотожно або отримана система рівнянь є несумісною. Система (3.1) є системою рівнянь у частинних похідних відносно $(n+1)^{2}=(2 m+1)^{2}$ невідомих функцій $\xi_{i}, \xi_{i, j}, \rho, \rho_{i}$. Вимога аналітичності контраваріантного поля $\xi^{i}$ дає $2 m^{2}$ обмежень. Остаточно отримані результати можна підсумувати у вигляді теореми.

Теорема 3.8. Для того, щоб ЛКK-многовид $\left(M^{n}, J, g\right)$ допускав наявність групи конформно голоморбно-проективних перетворень, необхідно та достатнъо, щоб система умов інтегровності (3.10), (3.13) та їх продовжень (3.14), (3.15), та ін., була сумісною. Тоді ЛККмноговид $\left(M^{n}, J, g\right)$ допускає наявність r-nараметричної групи, де

$$
r=2(m+1)^{2}-1-k,
$$

$a m i k \in$ відповідно комплексною розмірністю многовиду та рангом системи умов інтегровності, а також їх продовжень. У випадку, якщо умови (3.10) та (3.13) задоволъняються тотожно, розв'язок системи (3.1) залежатиме від $r=2(m+1)^{2}-1$ параметрів.

\section{9. Конформно голоморфно-проективні інфінітезимальні пе-} ретворення на компактних ЛКК-многовидах. Нехай $\left(M^{n}, J, g\right)$ $\epsilon$ компактним ЛКК-многовидом, а вектор $\xi$ породжує на цьому многовиді конформно голоморфно-проективні деформації (3.1). Згорнемо рівняння $(3.1)_{3} 3 g^{j k}$. Тоді отримаємо

$$
\begin{aligned}
\xi_{i, t}{ }^{t}-\xi_{\alpha} R_{i}^{\alpha} & =\frac{2-n}{2}\left(\omega_{\alpha} \xi^{\alpha}\right)_{, i}-\frac{\omega_{i}}{2} g^{j k} \mathfrak{L}_{\xi} g_{j k}+\frac{\omega^{\alpha}}{2}\left(\mathfrak{L}_{\xi} g_{i \alpha}\right) g_{j k} g^{j k} \\
& =\frac{2-n}{2}\left(\omega_{\alpha} \xi^{\alpha}\right)_{, i}+\frac{1}{2} \omega_{i} g_{j k} \mathfrak{L}_{\xi} g^{j k}+\frac{n}{2} \omega^{\alpha}\left(\mathfrak{L}_{\xi} g_{i \alpha}\right),
\end{aligned}
$$

або, піднімаючи у (3.16) індекс $i$,

$$
\xi_{, t}^{i t}-\xi^{\alpha} R_{\alpha}^{i}=\frac{(2-n) g^{i t}}{2}\left(\omega_{\alpha} \xi^{\alpha}\right)_{, t}+\frac{1}{2} \omega^{i} g_{j k} \mathfrak{L}_{\xi} g^{j k}-\frac{n}{2} \omega_{\alpha}\left(\mathfrak{L}_{\xi} g^{i \alpha}\right) .
$$

3 іншого боку, відомо [7], що для того, щоб контраваріантне векторне поле $\xi$ на компактному майже ермітовому многовиді було контраваріантним майже аналітичним, необхідно та достатньо, щоб тотожно виконувалась умова

$$
\xi_{, t}^{i t}-\xi^{\alpha} R_{\alpha}^{i}=-J_{\alpha}^{i}\left(\mathfrak{L}_{\xi} J_{\gamma, \beta}^{\beta} g^{\alpha \gamma}\right)+\frac{1}{2}\left(J_{k, j}^{\alpha}+J_{j, k}^{\alpha}\right) J_{\alpha}^{i} \mathfrak{L}_{\xi} g^{j k} .
$$

Для ЛКК-многовидів, враховуючи (2.7) та (2.6), маємо: 


$$
\begin{aligned}
-J_{\alpha}^{i}\left(\mathfrak{L}_{\xi} J_{\gamma, \beta}^{\beta} g^{\alpha \gamma}\right)+\frac{1}{2}\left(J_{k, j}^{\alpha}+J_{j, k}^{\alpha}\right) J_{\alpha}^{i} \mathfrak{L}_{\xi} g^{j k}= \\
\quad=\frac{(2-n) g^{i t}}{2}\left(\omega_{\alpha} \xi^{\alpha}\right)_{, t}+\frac{1}{2} \omega^{i} g_{j k} \mathfrak{L}_{\xi} g^{j k}-\frac{n}{2} \omega_{\alpha}\left(\mathfrak{L}_{\xi} g^{i \alpha}\right) .
\end{aligned}
$$

Порівнюючи (3.17) та (3.18), врахувавши (3.19), отримуємо теорему

Теорема 3.10. На компактному ЛКK-многовидi $\left(M^{n}, J, g\right)$ векторне поле $\xi$, що генеруе нетривіальні інфінітезимальні конформно голоморбно-проективні перетворення, є контраваріантним майэе аналітичним.

Також, відомо [6, с. 26], що будь який вектор на компактному многовиді має задовольняти рівності

$$
\int_{M^{n}}\left(\left(\xi_{, t}^{i t}-\xi^{\alpha} R_{\alpha}^{i}\right) \xi_{i}-\left(\mathfrak{L}_{\xi} g^{j k}\right)\left(\mathfrak{L}_{\xi} g_{j k}\right)-\left(\xi_{, t}^{t}\right)^{2}\right) d \sigma=0 .
$$

Враховуючи (3.20), з (3.17) отримуємо теорему.

Теорема 3.11. На компактному ЛКК-многовиді $\left(M^{n}, J, g\right)$ векторне поле $\xi$, що генеруе нетривіальні інфінітезимальні конформно голоморфно-проективні перетворення, має задовольняти умову

$$
\begin{aligned}
\int_{M^{n}}\left(\left(\frac{2-n}{2}\left(\omega_{\alpha} \xi^{\alpha}\right)_{, t}+\frac{1}{2} \omega_{i} g_{j k} \mathfrak{L}_{\xi} g^{j k}+\frac{n}{2} \omega^{\alpha}\left(\mathfrak{L}_{\xi} g_{i \alpha}\right)\right) \xi^{t}-\right. \\
\left.-\left(\mathfrak{L}_{\xi} g^{j k}\right)\left(\mathfrak{L}_{\xi} g_{j k}\right)-\left(\xi_{, t}^{t}\right)^{2}\right) d \sigma=0 .
\end{aligned}
$$

Зокрема, якщо

$$
\left(\frac{2-n}{2}\left(\omega_{\alpha} \xi^{\alpha}\right)_{, t}+\frac{1}{2} \omega_{i} g_{j k} \mathfrak{L}_{\xi} g^{j k}+\frac{n}{2} \omega^{\alpha}\left(\mathfrak{L}_{\xi} g_{i \alpha}\right)\right) \xi^{t}=0 \quad m a \quad \xi_{, t}^{t}=0,
$$

то інфінітезимальні перетворення породжувані ६ будуть рухами.

\section{ЛIтЕРАТУРА}

[1] Sorin Dragomir, Liviu Ornea. Locally conformal Kähler geometry, volume 155 of Progress in Mathematics. Birkhäuser Boston, Inc., Boston, MA, 1998.

[2] Josef Mikeš, Hana Chudá, Irena Hinterleitner. Conformal holomorphically projective mappings of almost Hermitian manifolds with a certain initial condition. Int. J. Geom. Methods Mod. Phys., 11(5):1450044, 8, 2014.

[3] Josef Mikeš, Elena Stepanova, Alena Vanžurová, et al. Differential geometry of special mappings. Palacký University Olomouc, Faculty of Science, Olomouc, 2015.

[4] Izu Vaisman. On locally conformal almost Kähler manifolds. Israel J. Math., 24(34):338-351, 1976. 
[5] Kentaro Yano. The theory of Lie derivatives and its applications. North-Holland Publishing Co., Amsterdam; P. Noordhoff Ltd., Groningen; Interscience Publishers Inc., New York, 1957.

[6] Kentaro Yano. Differential geometry on complex and almost complex spaces. International Series of Monographs in Pure and Applied Mathematics, Vol. 49. A Pergamon Press Book. The Macmillan Co., New York, 1965.

[7] Kentaro Yano, Mitsue Ako. Almost analytic vectors in almost complex spaces. Tôhoku Math. J. (2), 13:24-45, 1961.

[8] Б. А. Дубровин, С. П. Новиков, А. Т. Фоменко. Современная геометрия: методъ и приложенил. Наука. Гл. ред. физ.-мат. лит., 1986.

[9] В. Ф. Кириченко. Локально конформно-келеровы многообразия постоянной голоморфной секционной кривизны. Матем. сб., 182(3):354-363, 1991.

[10] В. Ф. Кириченко. Конформно-плоские локально конформно-келеровы многообразия. Матем. сб., 51(5):57-66, 1992.

[11] В. М. Кузаконь, В. Ф. Кириченко, О. О. Пришляк. Гладкі многовиди. Геометричні та топологічні аспекти, volume 97. Київ: Праці Інституту математики НАН України. Математика та ㄲï застосування, 2013.

[12] Й. Микеш. Голоморфно-проективные отображения и их обобщения. Геометрия 3, Итоги науки и техн. Сер. Соврем. мат. и ее прил. Темат. обз., 30:258-289, 2002.

[13] Є. В. Черевко. Голоморфно-проективні перетворення та конформно-келерові многовиди. Proc. Inter. Geom. Center, 4(1):51-64, 2016.

[14] Л. П. Эйзенхарт. Риманова геометрия. М.: ИЛ, 1948.

Черевко Євген Володимирович

ОДЕСЬКИЙ НАЦІОНАЛЬНИЙ ЕКОНОМІЧНИЙ УНІВЕРСИТЕТ, ОДЕСА, УКРАЇНА.

Email: cherevko@usa.com

Чепурна Олена Євгенівна

ОДЕСЬКИЙ НАЦІОНАЛЬНИЙ ЕКОНОМІЧНИЙ УНІВЕРСИТЕТ, ОДЕСА, УКРАЇНА.

Email: chepurna67@gmail.com 